\title{
Hippocampal Characteristics and Invariant Sequence Elements Distribution of GLRA2 and GLRA3 C-to-U Editing
}

\author{
Philipp Schaefermeier ${ }^{a, b}$ Sarah Heinze ${ }^{c-e}$ \\ ${ }^{a}$ Charité University Medicine Berlin, ${ }^{b}$ Helmholtz Group RNA Editing and Hyperexcitability Disorders, \\ Max-Delbrück-Centre for Molecular Medicine, and ' Institute of Forensic Sciences and Legal Medicine, \\ Charité Berlin, Berlin, ${ }^{\mathrm{d}}$ Klinikum Oldenburg gGmbH, Oldenburg, and ${ }^{\mathrm{e}}$ Institute of Forensic Medicine, \\ Johannes Gutenberg University of Mainz, Mainz, Germany
}

\section{Key Words}

Glycine receptor alpha subunit · GlyR $\cdot$ Mooring $\cdot$ RNA editing $\cdot$ Spacer $\cdot$ Temporal lobe epilepsy

\begin{abstract}
Glycine receptor a2 and a3 subunit (GLRA2/GLRA3) highaffinity variants, of which the subjacent amino acid substitutions issue from C-to-U RNA editing, are thought to influence tonic inhibition and pathophysiology. In light of the detection of GLRA3 NM_006529:r.1157C >U and GLRA2 NM_002063:r.1416C $>$ U exchanges in hippocampus explants of temporal lobe epilepsy patients, we now examine the healthy situation and relate it to the epileptic situation by ascertaining controls in a legitimate reanalysis. The GLRA2 and GLRA3 editing events that would ultimately result in a glycine receptor with increased affinity occur in the postmortem nonepileptic hippocampus. Most notably, their relative amounts do not significantly differ from those in increased damaged hippocampus explants, whereas curbed relative amounts in epileptic explants without cell loss come out statistically significant. Local sequence alignment reveals invariant sequence stretches consistent in GLRA2/ GLRA3 and other edited transcripts that coincide with known $A P O B$ sequence elements. Concerning the essential mooring element, GLRA2/GLRA3 comply strictly only with the motif's $5^{\prime}$ part. While this lack of canonical mooring elements and
\end{abstract}

uncertain action of the famous deaminase $A P O B E C 1$ suggest a specific regulation of GLRA2/GLRA3 editing, its reduction in the less-damaged epileptic hippocampus could be attributed to anomalous epileptic neurogenesis.

๑) 2016 S. Karger AG, Basel

Epilepsy, a major neurological disorder, is associated with a large number of syndromes all involving unpredictable seizures. About $36 \%$ of epileptic patients have inborn or acquired resistances to antiepileptic pharmaceuticals. Neurosurgery of an identified epileptic focus can provide remedy from seizures for highly affected patients, but novel medications are still sought for [Kwan and Brodie, 2000; Mohanraj and Brodie, 2006; Wood, 2012].

Typically, epileptogenic activities at the hippocampus would be balanced by its inhibitory neurons. A disruption of hippocampus interneurons can contribute to excitatory-inhibitory imbalance within the epileptic network [Sloviter, 1987; Nadler, 2003; Fröhlich et al., 2006; Reyes, 2006; Dudek and Sutula, 2007]. The glycine receptor (GlyR) is an inhibitory ligand-gated ion channel, like the $\mathrm{GABA}_{\mathrm{A}}$ receptor, although less abundant. It consists of an extracellular ligand-binding domain, a transmembrane chloride pore, formed by a pentameric setup of ligandbinding alpha subunits (GLRA1, GLRA2, GLRA3, also referred to as: GlyR $\alpha 1, \alpha 2, \alpha 3)$ with the structuring beta

\section{KARGER}

๑) 2016 S. Karger AG, Basel

E-Mail karger@karger.com

www.karger.com/msy
Philipp Schaefermeier

Poste restante

Kurfürstenstr. 2

DE-44147 Dortmund (Germany)

E-Mail pschaefe@ med.lmu.de 
subunit, and a rather variable intracellular domain [Langosch et al., 1990; Betz et al., 1999; Chattipakorn and McMahon, 2002; Keck et al., 2008]. GlyR is located inter alia in the hippocampus, in interneurons, at synaptic sites as well as extrasynaptically [Avila et al., 2013]. Modulation of excitatory postsynaptic potentials and inhibition in GABAergic interneurons via GlyR [Song et al., 2006] contributes to glycinergic tonic inhibition [Zhang et al., 2008; $\mathrm{Xu}$ and Gong, 2010].

Previously, a variant cDNA of GLRA3 (Glra3 NM_053724:r.701C $>$ U) that encodes a certain prolineto-leucine exchange (GLRA3 NP_446176:p.Pro234Leu; colloquially referred to as: GlyR a3 P185L) was described. The exchange alters a loop at the bottom of the ligandbinding domain adjacent to the brink of the pore. The variant subunit displays an increased apparent affinity for its ligand, likely enabling a high-affinity GlyR that responds to ambient glycine [Sherwin, 1999; Meier et al., 2005; Ogino et al., 2007; Du et al., 2015]. The corresponding substitution Glra2 NM_012568:r.1207C >U causes another high-affinity alpha subunit (GLRA2 NP_036700: p.Pro219Leu; colloquially referred to as: GlyR a2 P192L). In an analysis of hippocampus explants of pharmacoresistant temporal lobe epilepsy (TLE) patients, it was reported that the relative amounts of the same variations in humans, GLRA2 NM_002063:r.1416C $>\mathrm{U}$ and GLRA3 NM_006529:r.1157C >U (referred to as: GlyR a2 C575U and GlyR a 3 C554U), increase under severe sclerotic cell loss and secondary generalized tonic-clonic seizure (grand mal) frequency. However, no information on the nonepileptic state was given. Genomic GLRA2 DNA of patients carried no corresponding point mutation or single nucleotide polymorphism (SNP) [Eichler et al., 2008]. This supported the assumption of C-to-U RNA editing being responsible for the variants' origin, deduced from a sequence stretch similar to a spacer sequence downstream of Glra3 NM_053724:r.701C>U [Meier et al., 2005].

In $\mathrm{C}$-to-U-edited apolipoprotein $\mathrm{B}$ mRNA $(A P O B)$, the spacer and the mooring sequence elements are constituents of a stem loop that exposes a cytidine to enzymatic deamination. Several additional sequence elements, most important for $A P O B$ editing, flank the deaminated position from 60 nucleotides upstream to 160 nucleotides downstream [Shah et al., 1991; Backus and Smith, 1992; Driscoll et al., 1993; Hersberger and Innerarity, 1998; Sowden et al., 1998; Hersberger et al., 1999; Maris et al., 2005]. Two prominent proteins bind the $A P O B$ transcript: the cytidine deaminase apolipoprotein $B$ editing complex (APOBEC1) and the RNA-binding protein Apobec complementary factor. In the liver,
APOBEC1 deaminates a cytidine residue in the $A P O B$ transcript to a uridine residue, resulting in 2 protein variants, both physiologically relevant in energy metabolism [Teng et al., 1993; Chester et al., 2000; Lellek et al., 2000; Mehta et al., 2000; Henderson et al., 2001]. Until recently, few further C-to-U-edited transcripts had been characterized [Skuse et al., 1996; Wang et al., 2004]. Nowadays, numerous murine targets of APOBEC1 have been described, leading to further characterization of the mooring motif [Rosenberg et al., 2011].

In our study, postmortem hippocampus material is used to display the healthy situation of C-to-U edited human GLRA2 and GLRA3. By doing this, control groups are generated that augment the study mentioned above on epileptic hippocampus explants [Eichler et al., 2008]. Consequently, these results are presented alongside a legitimate reanalysis of the former series of experiments, adjusting the previous explanatory view on the relation of editing levels and course of disease. In addition, we describe the situation of several sequence elements and features most crucial to RNA editing in this matter.

\section{Materials and Methods}

\section{Quantitative Cloning Analysis}

Postmortem hippocampus tissue was obtained at autopsy from 3 individuals whose deaths had occurred out of hospital and were attributable to different natural and unnatural causes. Pre-existing pathological conditions of the central nervous system were ruled out by histology, and underlying intoxications were ruled out by toxicological analysis. Specimens were comparable to the TLE neurosurgery explant series in terms of their local origin, age, and fixationless procession [Eichler et al., 2008], except for their postmortem delays that exceeded the surgery explants for up to $5 \mathrm{~h}$. RNA was isolated with Trizol (Thermo Fisher, Waltham, MA, USA). Additionally, a commercially available pooled hippocampus RNA (provided by R.J. Harvey, London) was used [Rees et al., 2006]. Cloning assays to quantify GLRA2 NM_002063:r.1416C>U and GLRA3 NM_006529:r.1157C > U were performed in the identical setup described previously [Meier et al., 2005; Eichler et al., 2008]. Briefly, PCR used degenerated mismatch primers that introduce restriction sites only into templates with the respective nucleotide exchanges. After restriction endonuclease digestion, insertion into custom vectors, transformation, and determination of confirmed-positive colony numbers, the percentage of clones with a variant insert proportionate to the total amount of control clones reflected the relative amount of edited GLRA2/GLRA3 mRNA for each sample.

Instead of averaging triplicate measurements for one specimen and grouping the averages, all individual data points of each condition were grouped collectively. In postmortem GLRA2 measurements, 13/15 individual data points represent the 3 matched individuals, while $2 / 15$ data points represent the 20 pooled individuals. In the postmortem GLRA3, 6/8 data points represent the 3 matched
86

Mol Syndromol 2017;8:85-92

DOI: $10.1159 / 000453300$
Schaefermeier/Heinze 


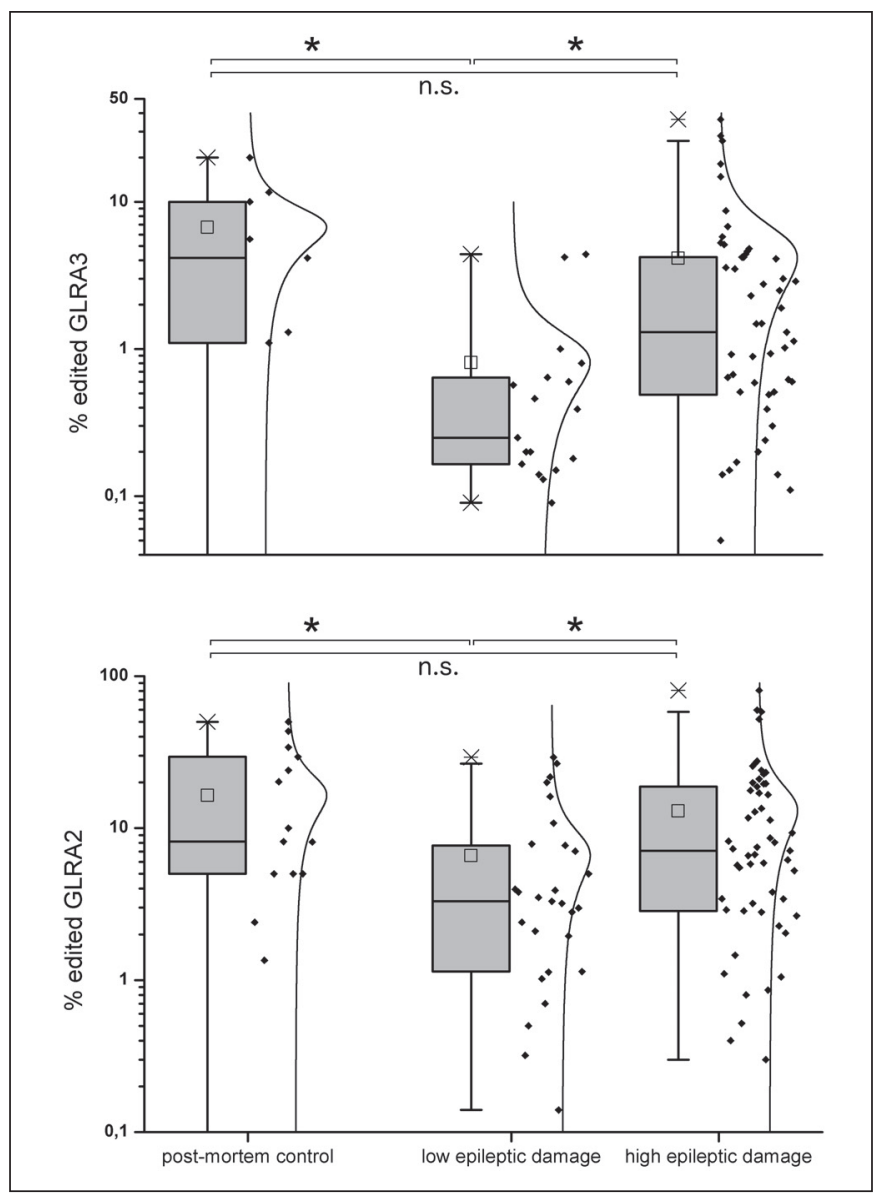

Fig. 1. The occurrence of GLRA2/GLRA3 C-to-U RNA editing in the healthy postmortem hippocampus is compared with reanalyzed corresponding data obtained from surgical explants of pharmacoresistant epilepsy patients [Eichler et al., 2008]. The relative amounts of edited GLRA2/GLRA3 (\% edited) in the healthy postmortem hippocampus versus the epileptic situation with increased damage (attributable to sclerotic cell loss and frequent grand mal) do not differ significantly. By contrast, the low relative amounts in epileptic explants without hippocampal cell loss and marked sclerosis are significant.

individuals, while $2 / 8$ data points represent the 20 pooled individuals. The approach allowed reanalyzing the dataset from formerly examined series of epilepsy surgery explants from pharmacoresistant TLE [Eichler et al., 2008], also including several specimens that had not yielded triplicate data. For comparability, general data grouping was retained. Seven individuals of the group of patients' explants without marked hippocampal cell loss and sclerosis are represented by 29 data points in GLRA2 measurements and by 18 data points in GLRA3. In the group with increased damages attributable to sclerosis, cell loss, and frequent grand mal, the 18 individuals are represented by 57 data points in GLRA2 measurements and by 53 data points in GLRA3. The Mann-Whitney test was performed with OriginPro 8G (OriginLab, Northampton, MA, USA), with a $p$ value less than 0.05 considered as statistically significant.
Database Coexpression Approach

Conserved domain database entries [Marchler-Bauer et al., 2015] with cytidine deaminase domains were examined for coexpression with GlyR in the hippocampus. GeneAtlas datasets in BioGPS gene reports were used to check for human brain expression of any genes of interest [Wu et al., 2009]. Murine in situ hybridization (ISH) data publicly available from the Allen Brain Atlas [Lein et al., 2007] were used to compare the hippocampal expression patterns of the candidate deaminase CDADC1 as well as GLRA2 and GLRA3. GLRA4 expression data served as negative control. Of each set of images (antisense probe, sagittal plane, experiment numbers: 70525807, CDADC1; 71587739, GLRA2; 70723453, GLRA3; 70724744, GLRA4) consisting of 19-20 sections, approximately the sixth (for the parasagittal plane) and thirteenth section (for the midsagittal plane) were selected, depending on pattern/plane visibility. To visualize the expression data in grayscale, the ISH and expression layers were transferred into the GIMP 2.8 raster graphics editor. Relevant expression domains were cropped. ISH layers were grayscaled, and brightness was set to +50 . The expression layers were inverted; the white color was replaced with transparent color, and all remaining colors were changed to black. Finally, both layers were merged.

\section{Sequence Comparison}

Nucleotide sequences of C-to-U-edited transcripts were traced from their respective GenBank entries. Sequence conservation not revealed by conventional comparison matrices was exposed via manual alignment with GeneDoc [Nicholas and Nicholas, 1997]. The hyphens indicate introduced gaps. The shading mode was set to conserved; primary conservation (black shading, uppercase consensus line) was set to $100 \%$; secondary conservation (dark gray shading, lowercase consensus line) was set to $75 \%$, and tertiary conservation (light gray shading, not marked in consensus line) was set to $40 \%$.

\section{Results}

\section{GLRA2 and GLRA3 Editing in the Hippocampus}

Pronounced relative amounts of the C-to- $\mathrm{U}$ editing events GLRA2 NM_002063:r.1416C>U and GLRA3 NM_006529:r.1157C >U occur in postmortem hippocampus RNA (Fig. 1), detected via quantitative cloning assays targeting the respective cDNA nucleotide exchanges.

Conjoint with equivalently reanalyzed epilepsy surgery explants, the postmortem series serves as nonepileptic controls. These data show comparatively smaller relative amounts of edited GLRA2/GLRA3 in explants without hippocampal cell loss than in both the postmortem control group and the group of explants with marked cell loss and grand mal frequency. The relative amounts of the 2 latter groups do not show significant differences (see n.s. in Fig. 1). The low of editing in the former group is statistically significant (asterisks in Fig. 1), attenuated in GLRA2, and more intensive in GLRA3. 


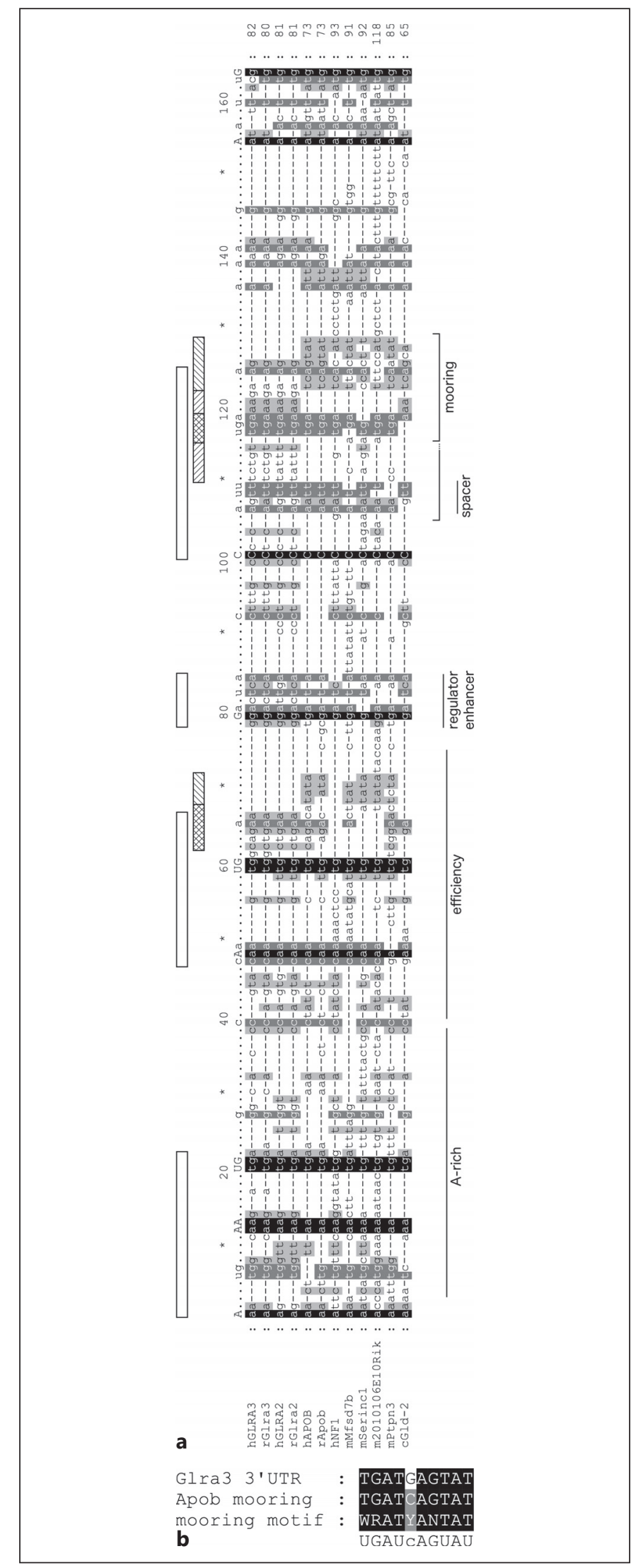

Consistent Stretches of Invariant Nucleotides, Shared in GLRA2 and GLRA3 and Other C-to-U-Edited

\section{Transcripts}

Using manual alignment of sequence subsections flanking the edited nucleotide, consistent sequence stretches of invariant and highly invariant nucleotides, shared between GLRA2/GLRA3 as well as in other C-to$\mathrm{U}$-edited transcripts and distributed in an interlaced pattern, were identified (Fig. 2a: viewed from $5^{\prime}$ to $3^{\prime}$, these consistent stretches appear in the "A....ug......AA.......UG" stretch located at marker position 1-23, the "cAa......... UG....a" stretch located at position 48-66, the "Ga.u.a" stretch at position 80-85, and the "C.......uu......uga" stretch located around position 101-118), each formed of up to 7 shared nucleotides. Another, more weakly shared stretch, that is not consistent in each inspected transcript, is "c.ga..tata", located at alignment position 63-80. Within the consistent stretches, variant or unique nucleotides appear, up to 5 or 9 in number. Outside of the consistent stretches, variant and unique nucleotides are present more often and in longer succession (see alignment positions 24-47, 86-100, and 130-154 in Fig. 2a).

The consistencies agree with sequence elements that have been demonstrated to be highly relevant for $A P O B$ editing: the "A....ug......AA......UG" stretch resides directly at the $5^{\prime}$ border of the upstream A-rich element determined in $A P O B$; the "Ga.u.a" stretch encloses the regulator sequence element; the "C.......uu.......uga" stretch comprises the well-known spacer and, in parts, the mooring element. GLRA2 and GLRA3 transcript sequences lack most of the $3^{\prime}$ and mid parts of the mooring motif, and they supplant it at position 120-124. In the $5^{\prime}$ part, they conform very well to the motif. Downstream of the moor-

Fig. 2. a Invariant nucleotides of GLRA2/GLRA3 shared with other C-to-U edited transcripts agree with known sequence elements. Rectangular bars indicate consistent stretches of invariant nucleotides. Hatched bars indicate weakly shared stretches, which are not consistent with all inspected transcripts (main diagonal hatching for consistencies in GLRA2/GLRA3 and few other transcripts, but not with $A P O B$; anti-diagonal hatching indicates consistencies in $A P O B$ and other transcripts, but not with GLRA2/GLRA3, and checkered hatching for consistencies in GLRA2/GLRA3 and $A P O B$, but not with every single one of the others). $A P O B$ sequence elements [Shah et al., 1991; Backus and Smith, 1992; Driscoll et al., 1993; Hersberger and Innerarity, 1998; Sowden et al., 1998; Hersberger et al., 1999] are indicated with lines at the bottom; mooring elements are indicated with hooked lines. Transcript prefixes indicate species. Deaminated cytidine at alignment position 100. b The 3' UTR of rat Glra3 (genebank entry M55250) contains a sequence nearly identical to the mooring element of Apob. Motif sequence from Rosenberg et al. [2011]. 
ing, another A-rich stretch is shared in all inspected transcripts. The 3 ' untranslated region (UTR) of rat Glra3 features an almost exact mooring sequence located 1,121 nucleotides downstream of the edited cytidine (Fig. 2b).

\section{Concurrent Features of CDADC1}

Apparent from database queries, CDADC1 possesses 2 cl00269 cytidine deaminase-like superfamily domains that resemble the catalytic domain of APOBEC1 (Fig. 3a, b). In murine hippocampus, there are overlaps in the gene expression patterns of $C d a d c 1$ in the dentate gyrus and in CA1-CA3 with those of Glra2 as well as in hilus with Glra3 (Fig. 4).

\section{Discussion}

Lately, one could follow a controversial debate on the physiologic extent of RNA editing, once again emphasizing the high level of detail necessary for its examination [Chakravarti, 2011; Li et al., 2011; Kleinman and Majewski, 2012; Wang et al., 2014]. Also the case of C-to-U-editing of GlyR alpha subunit RNA deserves thorough interpretation. After the original description, another study fit quite well to the feasible accuracy of detecting Glra3 NM_053724:r.701C>U, whereas it was omitted to detect native edited Glra3 transcripts [Meier et al., 2005; Nakae et al., 2008]. Later on, it was assumed that increased levels of editing were a specific attribute of the diseased epileptic state [Eichler et al., 2008; Winkelmann et al., 2014].

For the first time, this study describes distinct GLRA2 NM_002063:r.1416C>U and GLRA3 NM_006529: r.1157C > U RNA editing events in the healthy hippocampus using heterogenous postmortem material. This finding enabled our legitimate reanalysis of the previous series of experiments with the postmortem material integrated as necessary nonepileptic controls, showing the particular decline of the relative amounts of edited GLRA2 and GLRA3 in the epileptic hippocampus with lesser degrees of damage. This is a relevant result and refines accurate assessment of how epilepsy damages and editing levels are associated in pharmacoresistant TLE.

Ectopic granule neurons or anomalous latency phase neurogenesis [Parent et al., 1997, 2006; Scharfman and Pierce, 2012] may provide one explanation for the pathological decrease in editing and its different extent in the 2 subunit transcripts. Newly generated cells in the epileptic hippocampus can arise from more than one source area and can migrate in varying amounts towards DG and CA1/CA2 (the main expression domains of Glra2) as well as towards CA4 (the main region of Glra3 gene expression) [Crespel et al., 2005]. In general, young neurons show less GlyR gene expression [Aroeira et al., 2011], and rodent prenatal hippocampal neurons seldom display
Fig. 3. a Domain structure of CDADC1 compared with those of 2 paradigmatic Apobec family members. CDADC1 contains 2 domains that match the zinc-binding region of the cl00269 cytidine deaminase-like superfamily, to which the catalytic domain of the C-to- $U$ deaminase APOBEC1 also belongs. Modified after Marchler-Bauer et al. [2015]. b Sequence alignment of the similarities between CDADC1 and APOBEC1, within the cl00269 superfamily of cytidine and deoxycytidylate deaminases. The 2 deaminase domains of CDADC1 are abbreviated as dom 1 and dom2. cd01286, deoxycytidylate (dCMP) deaminase domain; pfam08210, APOBEC-like N-terminal (catalytic) domain; cd01283, cytidine deaminase zincbinding domain. Modified after MarchlerBauer et al. [2015].

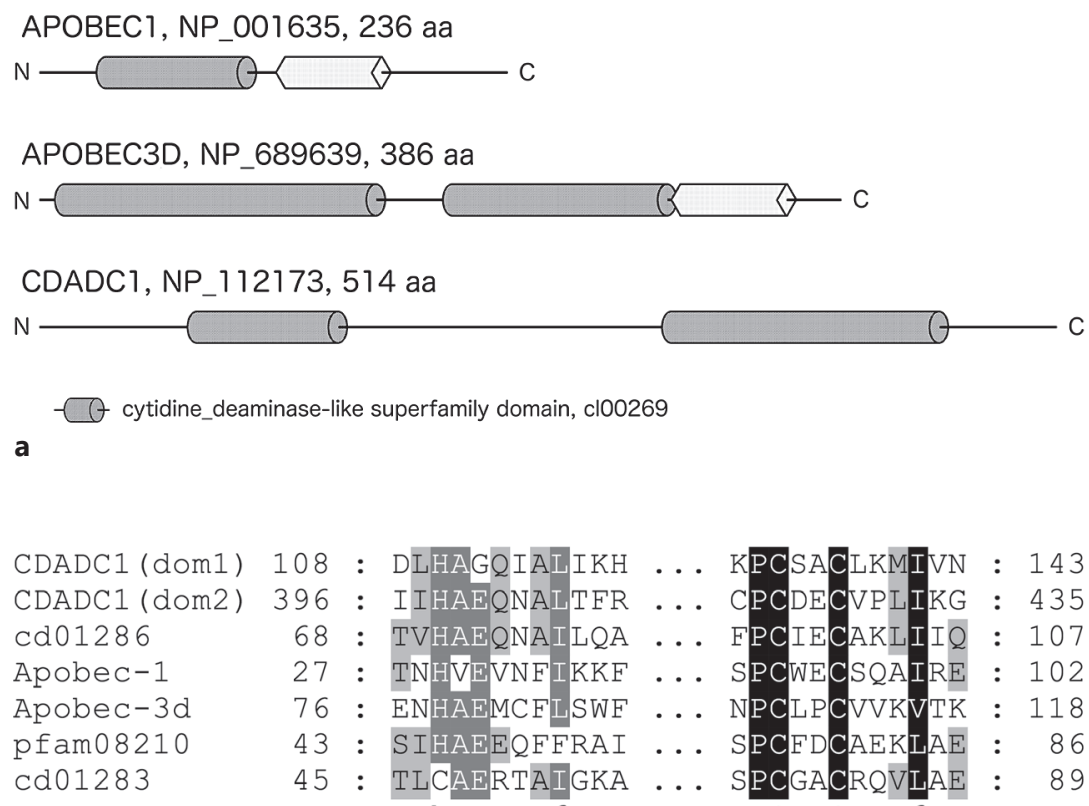


Fig. 4. Murine hippocampus gene expression patterns of Cdadc1 notably overlap with those of Glra2 in pyramidal layer of CA1-3 and in granule cell layer of dentate gyrus, with those of Glra3 in hilus or polymorph layer of dentate gyrus. Glra4 as negative control. Scale bars, parasagittal $500 \mu \mathrm{m}$; midsagittal $1000 \mu \mathrm{m}$. Modified after Lein et al. [2007].

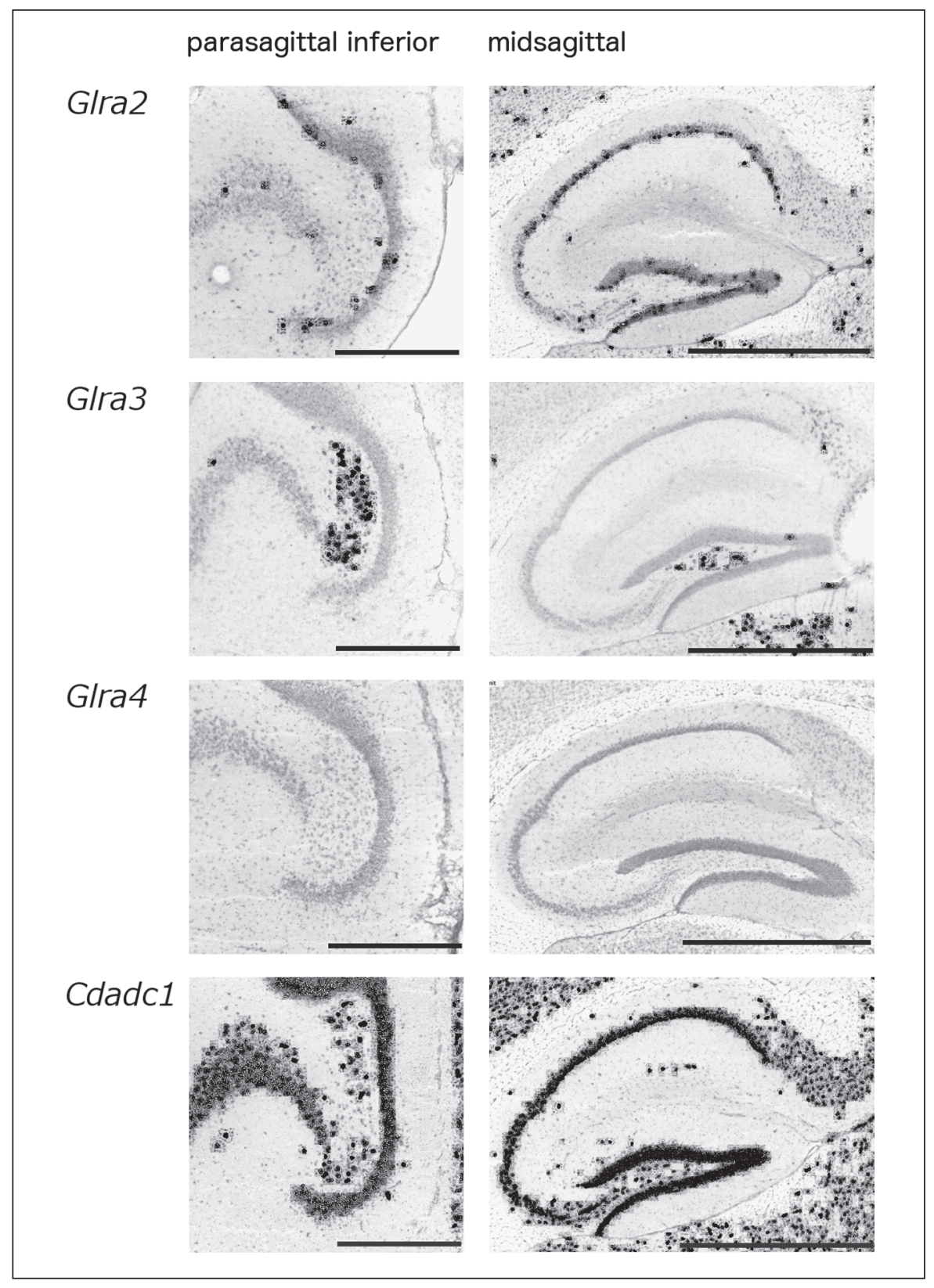

pronounced Glra2/Glra3 C-to-U events (unpubl. observation). Accordingly, a "dilution" of hippocampal cells by aberrant immature cells could be one possible cause of the apparent drop in editing. Sclerotic cell loss [Zhu et al., 1997; Blümcke et al., 2000, 2009] in the increased damaged explants group is not accompanied by significant reductions of C-to-U levels, compared to the nonepileptic postmortem situation, yet they could still play a role in the very slight reduction visible in this group.

Detecting editing events in PCR amplicons does not directly prove the existence of functional high-affinity
GlyR, but it tracks the activity of its deaminase, whose identity is another open problem. We present properties of CDADC1 that resemble those of the important cytidine deaminase APOBEC1 and of the concerned GLRA subunits in the hippocampus. In the brain, gene expression of $A P O B E C 1$ is relatively meagre (publicly available microarray data), and recent studies reported only very vaguely on its native expression in brain tissue [Gee et al., 2011; Guo et al., 2011; Papavasiliou et al., 2014]. Despite this apparent absence, silencing of glycinergic currents following steric inhibition of Apobec cytidine deaminas- 
es with a cytidine analogue had been reported [Meier et al., 2005]. Although the drug indeed can affect Apobecfamily deaminases, it should be argued that administering the cytidine analogue without sequence context could have influenced channel gating in different ways [Rausch et al., 2009; Seifert et al., 2011; Wedekind and Smith, 2012; Zong et al., 2012].

To determine the extent of the common sequence basis of well-characterized APOBEC1-mediated C-to-U editing with that of GLRA2/GLRA3, manual alignment of various $\mathrm{CDNA}$ sequences with documented $\mathrm{C}$-to- $\mathrm{U}$ editing events was performed. The consistent stretches of invariant nucleotides found accord with the upstream Arich, the regulator, and the spacer elements, which are very well characterized in Apob [Shah et al., 1991; Backus and Smith, 1992; Driscoll et al., 1993; Hersberger and Innerarity, 1998; Sowden et al., 1998; Hersberger et al., 1999]. The discovery of additional murine targets of APOBEC1 had led to further characterization of the mooring element [Rosenberg et al., 2011], but GlyR a2 and $\alpha 3$ transcript sequences fit this motif only in its $5^{\prime}$ part (it should be noted that the seemingly merged spacer and mooring elements of Serinc1 also deviate from the motif). Whether the conventional mooring in the $3^{\prime}$ UTR of rat Glra3 corresponds to a respective C-to- $\mathrm{U}$ event is not known. Many of the additional murine transcripts carry their edited positions and their mooring elements in the 3' UTR [Rosenberg et al., 2011], whereas in transcripts encoding GLRA2/GLRA3, the edited positions and their adjacent $3^{\prime}$ deviant mooring elements are present in the open reading frame (ORF). A mooring sequence in a UTR can understandably comply with the motif more closely than in an ORF, where mandatory compliance to codon information restricts sequence variability.

In this regard, 2 known synonymous SNPs in the inspected sequence section of GLRA3 should be mentioned: Rs140655344 shifts a glutamate codon from GAG to GAA, making the A-rich region more A-rich. Rs41279513 can appear in the spacer, changing a glutamine codon from CAG to CAA, creating a spacer element identical to that of $A P O B$. While invariant nucleotides could participate in secondary structures that capacitate binding or efficacy of a deaminase complex [Hersberger et al., 1999; Maris et al., 2005], deviations from the established elements could possibly even condition an unusual deaminase setup.

\section{Acknowledgments}

The authors wish to acknowledge the participation of Jochen C Meier (Technical University, Braunschweig), Thomas-Nicolas Lehmann (Helios Clinics, Bad Saarow), Michael Tsokos (Charité University Medicine, Berlin), and Sandeep S Amin (Klinikum Oldenburg, Oldenburg).

\section{Statement of Ethics}

Ethical approval for the conducted experiments was under Charité EA 1/142/05.

\section{Disclosure Statement}

The authors have no conflicts of interest to declare.

\section{References}

Aroeira RI, Ribeiro JA, Sebastião AM, Valente CA: Age-related changes of glycine receptor at the rat hippocampus: from the embryo to the adult. J Neurochem 118:339-353 (2011).

Avila A, Nguyen L, Rigo JM: Glycine receptors and brain development. Front Cell Neurosci 7:184 (2013)

Backus JW, Smith HC: Three distinct RNA sequence elements are required for efficient apolipoprotein B (apoB) RNA editing in vitro. Nuc Acid Res 20:6007-6014 (1992).

Betz H, Kuhse J, Schmieden V, Laube B, Kirsch J, Harvey RJ: Structure and functions of inhibitory and excitatory glycine receptors. Ann NY Acad Sci 868:667-676 (1999).

Blümcke I, Suter B, Behle K, Kuhn R, Schramm J, et al: Loss of hilar mossy cells in Ammon's horn sclerosis. Epilepsia 41 Suppl 6:S174-80 (2000).

Blümcke I, Kistner I, Clusmann H, Schramm J, Becker AJ, et al: Towards a clinico-pathologi- cal classification of granule cell dispersion in human mesial temporal lobe epilepsies. Acta Neuropathol 117:535-544 (2009).

Chakravarti A: Widespread promiscuous genetic information transfer from DNA to RNA. Circ Res 109:1202-1203 (2011).

Chattipakorn SC, McMahon LL: Pharmacological characterization of glycine-gated chloride currents recorded in rat hippocampal slices. J Neurophysiol 87:1515-1525 (2002).

Chester A, Scott J, Anant S, Navaratnam N: RNA editing: cytidine to uridine conversion in apolipoprotein B mRNA. Biochim Biophys Acta 1494:1-13 (2000).

Crespel A, Rigau V, Coubes P, Rousset MC, de Bock F, et al: Increased number of neural progenitors in human temporal lobe epilepsy. Neurobiol Dis 19:436-450 (2005).

Driscoll DM, Lakhe-Reddy S, Oleksa LM, Martinez D: Induction of RNA editing at heterolo- gous sites by sequences in apolipoprotein B mRNA. Mol Cell Biol 13:7288-7294 (1993).

Du J, Lü W, Wu S, Cheng Y, Gouaux E: Glycine receptor mechanism elucidated by electron cryo-microscopy. Nature 526:224-229 (2015).

Dudek FE, Sutula TP: Epileptogenesis in the dentate gyrus: a critical perspective. Prog Brain Res 163:755-773 (2007)

Eichler S, Kirischuk S, Jüttner R, Schaefermeier $\mathrm{PK}$, Legendre P, et al: Glycinergic tonic inhibition of hippocampal neurons with depolarizing GABAergic transmission elicits histopathological signs of temporal lobe epilepsy. J Cell Mol Med 12:2848-2866 (2008).

Fröhlich F, Timofeev I, Sejnowski TJ, Bazhenov M: Extracellular potassium dynamics and epileptogenesis, in Soltesz I, Staley K (eds): Computational Neuroscience in Epilepsy, ed 1, pp 435-437 (Elsevier, London 2006). 
Gee P, Ando Y, Kitayama H, Yamamoto SP, Kanemura Y, et al: APOBEC1-mediated editing and attenuation of herpes simplex virus 1 DNA indicate that neurons have an antiviral role during herpes simplex encephalitis. J Virol 85:9726-9736 (2011).

Guo JU, Su Y, Zhong C, Ming GL, Song H: Hydroxylation of 5-methylcytosine by TET1 promotes active DNA demethylation in the adult brain. Cell 145:423-434 (2011).

Henderson JO, Blanc V, Davidson NO: Isolation, characterization and developmental regulation of the human apobec-1 complementation factor $(A C F)$ gene. Biochim Biophys Acta 1522:22-30 (2001).

Hersberger M, Innerarity TL: Two efficiency elements flanking the editing site of cytidine 6666 in the apolipoprotein B mRNA support mooring-dependent editing. J Biol Chem 273: 9435-9442 (1998).

Hersberger M, Patarroyo-White S, Arnold KS, Innerarity TL: Phylogenetic analysis of the apolipoprotein B mRNA-editing region. Evidence for a secondary structure between the mooring sequence and the $3^{\prime}$ efficiency element. J Biol Chem 274:34590-34597 (1999).

Keck T, Lillis KP, Zhou Y, White JA: Frequencydependent glycinergic inhibition modulates plasticity in hippocampus. J Neurosci 28: 7359-7369 (2008).

Kleinman CL, Majewski J: Comment on "Widespread RNA and DNA sequence differences in the human transcriptome". Science 335: 1302, author reply 1302 (2012).

Kwan P, Brodie MJ: Early identification of refractory epilepsy. N Engl J Med 342:314-319 (2000).

Langosch D, Becker CM, Betz H: The inhibitory glycine receptor: a ligand-gated chloride channel of the central nervous system. Eur J Biochem 194:1-8 (1990).

Lein ES, Hawrylycz MJ, Ao N, Ayres M, Bensinger A, et al: Genome-wide atlas of gene expression in the adult mouse brain. Nature 445: 168-176 (2007).

Lellek H, Kirsten R, Diehl I, Apostel F, Buck F, Greeve J: Purification and molecular cloning of a novel essential component of the apolipoprotein B mRNA editing enzyme-complex. J Biol Chem 275:19848-19856 (2000).

Li M, Wang IX, Li Y, Bruzel A, Richards AL, et al: Widespread RNA and DNA sequence differences in the human transcriptome. Science 333:53-58 (2011).

Marchler-Bauer A, Derbyshire MK, Gonzales NR, Lu S, Chitsaz F, et al: CDD: NCBI's conserved domain database. Nucleic Acids Res 43(Database issue):D222-226 (2015).

Maris C, Masse J, Chester A, Navaratnam N, Allain FH: NMR structure of the apoB mRNA stem-loop and its interaction with the $\mathrm{C}$ to $\mathrm{U}$ editing APOBEC1 complementary factor. RNA 11:173-186 (2005).

Mehta A, Kinter MT, Sherman NE, Driscoll DM: Molecular cloning of apobec- 1 complementation factor, a novel RNA-binding protein involved in the editing of apolipoprotein $\mathrm{B}$ mRNA. Mol Cell Biol 20:1846-1854 (2000).

Meier JC, Henneberger C, Melnick I, Racca C, Harvey RJ, et al: RNA editing produces gly- cine receptor $\alpha 3^{\mathrm{P} 185 \mathrm{~L}}$ resulting in high agonist potency. Nat Neurosci 8:736-744 (2005).

Mohanraj R, Brodie MJ: Diagnosing refractory epilepsy: response to sequential treatment schedules. Eur J Neurol 13:277-282 (2006).

Nadler JV: The recurrent mossy fiber pathway of the epileptic brain. Neurochem Res 28:16491658 (2003).

Nakae A, Tanaka T, Miyake K, Hase M, Mashimo $\mathrm{T}$ : Comparing methods of detection and quantitation of RNA editing of rat glycine receptor alpha3 ${ }^{\text {P185L }}$. Int J Biol Sci 4:397-405 (2008).

Nicholas KB, Nicholas HB: GeneDoc: a tool for editing and annotating multiple sequence alignments. Distributed by the author (1997). Available from: http://www.nrbsc.org/gfx/ genedoc/gdsrc.htm.

Ogino S, Gulley ML, den Dunnen JT, Wilson RB; Association for Molecular Patholpogy Training and Education Committtee: Standard mutation nomenclature in molecular diagnostics: practical and educational challenges. J Mol Diagn 9:1-6 (2007).

Papavasiliou N, Chung Y, Gagnidze K, Gal-Toth J, Rayon V, Bulloch K: APOBEC1-mediated RNA editing in microglia and its consequences for inflammation in the brain (poster presentation). Ann Meet Society for Neuroscience, Chicago 2014.

Parent JM, Yu TW, Leibowitz RT, Geschwind DH, Sloviter RS, Lowenstein DH: Dentate granule cell neurogenesis is increased by seizures and contributes to aberrant network reorganization in the adult rat hippocampus. J Neurosci 17:3727-3738 (1997).

Parent JM, Elliott RC, Pleasure SJ, Barbaro NM, Lowenstein DH: Aberrant seizure-induced neurogenesis in experimental temporal lobe epilepsy. Ann Neurol 59:81-91 (2006).

Rausch JW, Chelico L, Goodman MF, Le Grice SFJ: Dissecting APOBEC3G substrate specificity by nucleoside analog interference. J Biol Chem 284:7047-7058 (2009).

Rees MI, Harvey K, Pearce BR, Chung SK, Duguid IC, et al: Mutations in the gene encoding GlyT2 (SLC6A5) define a presynaptic component of human startle disease. Nat Genet 38: 801-806 (2006).

Reyes AD: Experimental and theoretical analyses of synchrony in feedforward Networks, in Soltesz I, Staley K (eds): Computational Neuroscience in Epilepsy, ed 1, pp 304-315 (Elsevier, London 2006).

Rosenberg BRR, Hamilton CE, Mwangi MM, Dewell S, Papavasiliou NF: Transcriptomewide sequencing reveals numerous APOBEC1 mRNA-editing targets in transcript $3^{\prime}$ UTRs. Nat Struct Mol Bio 18:230-236 (2011).

Scharfman HE, Pierce JP: New insights into the role of hilar ectopic granule cells in the dentate gyrus based on quantitative anatomic analysis and three-dimensional reconstruction. Epilepsia 53 Suppl 1:109-115 (2012).

Seifert R, Beste K, Burhenne H, Voigt U, Wolter $S$, et al: Cyclic CMP and cyclic UMP: new (old) second messengers. BMC Pharmacol 11 Suppl 1:O34 (2011).

Shah RR, Knott TJ, Legros JE, Navaratnam N, Greeve JC, Scott J: Sequence requirements for the editing of apolipoprotein B mRNA. J Biol Chem 266:16301-16304 (1991).

Sherwin AL: Neuroactive amino acids in focally epileptic human brain: a review. Neurochem Res 24:1387-1395 (1999).

Skuse GR, Cappione AJ, Sowden M, Metheny LJ, Smith HC: The neurofibromatosis type I messenger RNA undergoes base-modification RNA editing. Nucleic Acids Res 24:478-485 (1996).

Sloviter RS: Decreased hippocampal inhibition and a selective loss of interneurons in experimental epilepsy. Science 235:73-76 (1987).

Song W, Chattipakorn SC, McMahon LL: Glycine-gated chloride channels depress synaptic transmission in rat hippocampus. J Neurophysiol 95:2366-2379 (2006).

Sowden MP, Eagleton MJ, Smith HC: Apolipoprotein B RNA sequence $3^{\prime}$ of the mooring sequence and cellular sources of auxiliary factors determine the location and extent of promiscuous editing. Nucleic Acids Res 26:16441652 (1998).

Teng B, Burant CF, Davidson NO: Molecular cloning of an apolipoprotein $\mathrm{B}$ messenger RNA editing protein. Science 260:1816-1819 (1993).

Wang IX, Core LJ, Kwak H, Brady L, Bruzel A, et al: RNA-DNA differences are generated in human cells within seconds after RNA exits polymerase II. Cell Rep 6:906-915 (2014).

Wang L, Kimble J, Wickens M: Tissue-specific modification of gld-2 mRNA in C. elegans: likely C-to-U editing. RNA 10:1444-1448 (2004).

Wedekind JE, Smith HC: Content dependent inhibitors of cytidine dreaminess and uses thereof. US Patent No 8158770 B2 (2012).

Winkelmann A, Maggio N, Eller J, Caliskan G, Semtner M, et al: Changes in neural network homeostasis trigger neuropsychiatric symptoms. J Clin Invest 124:696-711 (2014).

Wood H: Epilepsy: determinants of quality of life in epilepsy go beyond seizure-related variables. Nat Rev Neurol 8:59 (2012).

Wu C, Orozco C, Boyer J, Leglise M, Goodale J, et al: BioGPS: an extensible and customizable portal for querying and organizing gene annotation resources. Genome Biol 10:R130 (2009).

Xu TL, Gong N: Glycine and glycine receptor signaling in hippocampal neurons: diversity, function and regulation. Prog Neurobiol 91: 349-361 (2010).

Zhang LH, Gong N, Fei D, Xu L, Xu TL: Glycine uptake regulates hippocampal network activity via glycine receptor-mediated tonic inhibition. Neuropsychopharmacology 33:701-711 (2008).

Zhu ZQ, Armstrong DL, Hamilton WJ, Grossman RG: Disproportionate loss of CA4 parvalbumin-immunoreactive interneurons in patients with Ammon's horn sclerosis. J Neuropathol Exp Neurol 56:988-998 (1997).

Zong X, Krause S, Chen CC, Krüger J, Gruner C, et al: Regulation of hyperpolarization-activated cyclic nucleotide-gated (HCN) channel activity by cCMP. J Biol Chem 287:2650626512 (2012). 